\title{
RATIONAL DESIGN OF ANTIVIRAL DRUG COMBINATIONS BASED ON EQUIPOTENCY USING HCV SUBGENOMIC REPLICON AS AN IN VITRO MODEL
}

Mohamed Mandour ${ }^{1,2^{\star}}$, Inge Vliegen ${ }^{1, *}$, Jan Paeshuyse ${ }^{3, \#}$ and Johan Neyts ${ }^{1}$

${ }^{1}$ Rega Institute for Medical Research, University of Leuven (KU Leuven), B-3000 Leuven, Belgium

${ }^{2}$ Department of Clinical Pathology, Faculty of Medicine, Suez Canal University, Ismailia, Egypt.

${ }^{3}$ KU Leuven, Department of Biosystems, Division Animal and Human Health Engineering, Host Pathogen Interactions, B-3001 Leuven, Belgium

*Both authors contributed equally

${ }^{\#}$ Corresponding author:

Mailing address: Department of Biosystems, Division Animal and Human Health Engineering, Kasteelpark Arenberg 30 - bus 2456, 3001 Leuven. Phone: +32 163746 54. Fax: +32 16321994 . Email: Jan.Paeshuyse@kuleuven.be 


\section{Abstract :}

Combination therapy of directly acting antivirals (DAA's) for the treatment of chronic HCV infections has proven to be a highly effective strategy to cure chronic infections with this virus. Here we studied, using $\mathrm{HCV}$ as an example, how to best design in vitro studies that explore the combined antiviral efficiency of combinations of three or more DAA's. To that end we used a HCV NS3 protease inhibitor, a NS5A targeting compound and two non-nucleoside NS5B polymerase inhibitors (each one targeting a different drug binding site). We demonstrate, employing HCV subgenomic replicon containing Huh 9-13 hepatoma cells, that quadruple therapy with these 4 different DAA's each at $1 x$ their $E C_{75}$, results in a highly efficient inhibition of viral replication. This is further reflected in the rapid clearance of the HCV replicon from the host cell. By contrast, neither equipotent combinations that consist of either molecules alone at $4 \mathrm{x} \mathrm{EC}_{75}$ nor triple combinations at $1.33 \mathrm{x}$ the $\mathrm{EC}_{75}$ resulted in clearance. In contrast to the quadruple combo, drug-resistant variants emerged under mono-treatment and in most triple combo's. These data thus demonstrate that quadruple combinations at total suboptimal concentrations [i.e. concentrations at which neither mono- nor triple therapy is sufficiently potent] result rapidly in a pronounced antiviral efficacy. Altogether, this work provides an example as to how to design studies to explore the antiviral efficacy of combinations of more than two compounds.

\section{Keywords: Antiviral, Equipotent Combinations, HCV, DAA, Drug resistance}


Hepatitis C virus (HCV), is a major cause of chronic liver disease including liver cirrhosis, liver failure and hepatocellular carcinoma (HCC) (Oze et al., 2013, Kwong et al., 2011). In 2011, the first-generation of direct acting antiviral (DAA) agents, telaprevir (TLV) and boceprevir (BCV), were approved for the treatment of chronic HCV infections, rapidly followed in the years thereafter by the next generation of DAA's and combinations thereof. Currently available DAA combination regimens typically offer SVR rates well over 95\% (Li \& De Clercq, 2017).

We here made use of HCV (subgenomic replicons) as a model to rationally approach the design of experiments to study the antiviral efficacy of combinations of more than two compounds. We explored the efficacy of different quadruple combinations of DAA's, i.e. an HCV NS3 protease inhibitor, a NS5A inhibitor and two non-nucleoside polymerase inhibitors [targeting different drug binding sites of this enzyme] to clear hepatoma cells from their replicon without emergence of drug-resistant variants. To this end we made use

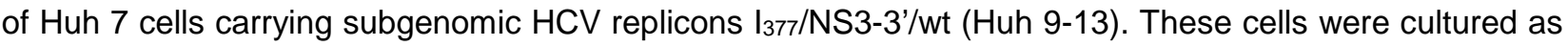
described before (Delang et al., 2011, Vliegen et al., 2009). The following molecules were included in this study: ITMN-191 (Danoprevir, NS3 protease inhibitor), BMS-790052 (Daclatasvir, NS5A inhibitor), VX-222 (NS5B thumb site II inhibitor) and GS-9190 (tegobuvir, NS5B palm-site inhibitor). Antiviral and cytostatic assays as well as clearance-rebound experiments were performed as described before (Vliegen et al., 2009). Sequencing of replicon populations was performed as described before (Delang et al., 2011).

First, we defined an equipotent concentration as the concentration of a specific antiviral that results in the same level or percentage of inhibition, i.e. $\mathrm{EC}_{6}, \mathrm{EC}_{13}, \mathrm{EC}_{25}, \mathrm{EC}_{50}$ and $\mathrm{EC}_{75}$-concentrations. Next, doseresponse curves were generated for the HCV inhibitors danoprevir, daclatasvir, VX-222 and tegobuvir in Huh 9-13 replicon containing cells and $\mathrm{EC}_{6}, \mathrm{EC}_{13}, \mathrm{EC}_{25}, \mathrm{EC}_{50}$ and $\mathrm{EC}_{75}$ were calculated for each individual DAA. Thereafter, quadruple and triple drug combinations at their respective concentration that results in equipotent levels of inhibition (i.e. each molecule respectively at its $\mathrm{EC}_{6}, \mathrm{EC}_{13}, \mathrm{EC}_{25}, \mathrm{EC}_{50}$ or $\mathrm{EC}_{75}$ concentration) were set-up. Their antiviral activity, as assessed in a 3-day assay, was compared with that of their theoretically predicted antiviral efficacy (Table 1). The theoretical predicted \% HCV inhibition is calculated for a specific quadruple combination of drugs at a given $\mathrm{x} \%$ effective concentration (ECX) by the equation $\mathrm{A}=\mathrm{B}+\mathrm{C}+\mathrm{D}+\mathrm{E}$, in which $\mathrm{A}$ represent the theoretically predicted $\% \mathrm{HCV}$ inhibition, $\mathrm{B}=\mathrm{x}, \mathrm{C}=$ $(100-B)^{\star} x^{*} 10-2, \quad D=(100-(B+C))^{*} x^{*} 10-2$ and $E=(100-(B+C+D))^{*} x^{\star} 10-2$. In the case of triple drug combinations $E=0$. The in vitro antiviral activity of both triple and quadruple equipotent mixtures of DAAs 
was determined to be more or less comparable to the theoretically calculated additive antiviral activity. Hence, the antiviral activities of the different combinations in this 3-day antiviral assay can thus be considered as additive. This experiment provides however only information about the antiviral efficacy of the combinations during short-term antiviral assays. When exploring the use of combinations for the treatment of chronic infections it will however be important to assess the antiviral potency and the possible emergence of resistant variants over longer periods of time. It should be noted that the concentrations of the compound used in this study are likely well below the concentrations in the (human) liver. When taking the NS3 protease inhibitor danoprevir as an example, liver concentrations in rats and cynomolgus monkeys were found to be much higher (Seiwert et al., 2008) than the in vitro $\mathrm{EC}_{50}$ or even $\mathrm{EC}_{99}$.

To that end an experimental set-up was designed to directly compare the efficacy of mono-, triple and quadruple therapy using fixed equipotent concentrations. For example the efficacy of a single compound at $4 \mathrm{x}$ it's $\mathrm{EC}_{75}$ is compared to that of a quadruple combination whereby each compound is used at $1 \mathrm{x}$ it's $\mathrm{EC}_{75}$ or a triple combination whereby each compound is used at 1.33 x it's $E_{75}$. Mathematically, such equipotent combinations should have the same level of antiviral activity if each compound is considered to have the same biological characteristics. The experiment was designed such that the potential clearance of the replicon from the host cell, or the emergence of drug-resistant variants, was monitored for multiple passage over a time-span of several weeks. Replicon RNA was quantified by means of RT-qPCR. The clearance rebound assay was designed as depicted in Figure 1. In brief, during the consecutive clearance passages of HCV subgenomic replicon containing cells, antiviral treatment will result in inhibition of replicon replication. This in turn results in reduced levels of replicon RNA copies, that is paralleled by reduced translation of replicon encoded proteins, including the neomycin-phosphotransferase gene (Neo). During the rebound passages the antiviral inhibitors are no longer present, as a consequence replication can resume and subgenomic replicon containing cells are selected by the addition of G418. Only cells that express sufficient quantities of the Neo-gene encoded by the replicon will survive the selective pressure of the added G418. The replicons of cells that survived the rebound were genotyped so as to assess whether resistant variants emerged during the consecutive clearance passages.

Treating Huh 9-13 replicon containing cells with a quadruple combination of each DAA at $1 x$ their $\mathrm{EC}_{6}$ concentration resulted in only a minor decrease (maximally $0.7 \log _{10}$ ) in viral RNA content (Figure 2 ). A quadruple combination of each DAA at $1 x$ their $\mathrm{EC}_{75}$ resulted in a decrease in viral RNA content of $4.5 \log _{10}$ at passage 6 (Figure 2). Monotherapy at equipotent concentrations (4x EC 75 of each single DAA) resulted 
in a maximum $3.8 \log _{10}$ reduction in viral RNA levels (i.e. danoprevir and tegobuvir) (Figure 3). By contrast even when DAA's were given as monotherapy at $10 x \mathrm{EC}_{75}$ viral breakthrough with the appearance of resistance mutations was observed for all inhibitors studied. Although a comparable maximal reduction in viral RNA content was observed for quadruple combination (with each compound at its $\mathrm{EC}_{75}$ or $\mathrm{EC}_{50}$ ), and triple drug combination (with each compound at $1.33 x$ their $\mathrm{EC}_{75}$ or $\mathrm{EC}_{50}$ ) the rate of this reduction were slower for the triple combination compared to the quadruple combination (Figures 2 and 4).

Quadruple combinations of each molecule at $1 \mathrm{x}$ their $\mathrm{EC}_{75}$ cured the hepatoma cells from their replicon already after two passages (6 days) of antiviral pressure. Indeed, when these cultures were further passaged in the presence of G418 none survived, indicating that there was no (or insufficient) replicon left to allow survival of the cells in the presence of G418 selective pressure. Quadruple combinations of each molecule at lower concentrations ( $E_{50}$ concentration or lower $\mathrm{EC}_{\mathrm{x}}$ concentrations) were however not able to completely clear the Huh 9-13 cells from their replicons. Genotyping of replicons carried by cells that had been treated with the quadruple combination and that had survived the rebound phase did, interestingly, not reveal mutations in the $\mathrm{HCV}$ replicons. By contrast, concentrations of $4 \mathrm{x} \mathrm{EC}_{75}$ of each individual compound alone did not clear cells from their replicon, even not following 6 passages; also drug resistant variants were detected in most of the replicons isolated from these cultures. Resistance mutations were observed when replicon-containing cells were treated with triple combinations of each compound (danoprevir, daclastavir and tegobuvir) at either their $\mathrm{EC}_{50}$ or $\mathrm{EC}_{75}$ concentration (Table 2). Cells surviving 4 passages of treatment with the $E_{75}$ triple combination of each compound at the $E_{75}$ resulted in the outgrowth of cells that carried replicons with signature mutations of tegobuvir and danoprevir resistance (Table 2). A comparison of triple and quadruple combinations versus the use of a single compound, all being equipotent, (Figures 2-4) revealed that an initial decrease brought about by the quadruple combination of $\geq 3 \log _{10}$ followed by a further gradual decrease in RNA content at later passages, resulted in total replicon clearance.

The use of combinations of DAA's for the treatment of chronic HCV infections has proven to be a highly effective strategy to cure chronic infections with this virus (Li \& De Clercq, 2017). Here we explored, using $\mathrm{HCV}$ as an example, how to best design in vitro studies that (i) explore the combined efficiency of three or more antiviral molecules and (ii) allow to simultaneously monitor the potential selection of drug-resistant variants. We demonstrate in this particular experimental setting that quadruple treatment with 4 different HCV DAA's is highly efficient in achieving a rapid cure of the cells from their viral replicon andthat total equipotent concentrations of either molecule alone or equipotent concentrations of triple combinations are 
not able to reach the same efficacy. Many studies have explored the combined efficacy of two antiviral agents, however, there is little literature on systematic studies in which the efficacy of triple or quadruple combinations is explored. The experimental design proposed here will provide guidance when designing future multiple combination studies.

\section{Acknowledgments}

The authors appreciate the fine technical assistance of Katrien Geerts and editorial assistance of Kim Donckers, Dominique Brabants and Christiane Callebaut. M. Mandour received a postdoctoral fellowship from the Egyptian Ministry of Higher Education and Scientific Research.

\section{References}

Delang L, Vliegen I, Froeyen M, Neyts J. Comparative study of the genetic barriers and pathways towards resistance of selective inhibitors of hepatitis $C$ virus replication. Antimicrob. Agents Chemother. 2011, 55, 4103-4113.

Kwong AD, Kauffman RS, Hurter P, Mueller P. Discovery and development of telaprevir: An NS3-4A protease inhibitor for treating genotype 1 chronic hepatitis C virus. Nat. Biotechnol. 2011, 29, 993-1003.

Li G, De Clercq E. Current therapy for chronic hepatitis C: The role of direct-acting antivirals. Antiviral Res. 2017, 142, 83-122.

Oze T, Hiramatsu N, Mita E, Akuta N, Sakamoto N, Nagano H, Itoh Y, Kaneko S, Izumi N, Nomura H, Hayashi N, Takehara T. A multicenter survey of re-treatment with pegylated interferon plus ribavirin combination therapy for patients with chronic hepatitis C in Japan. Hepatol. Res. 2013, 43, 35-43.

Seiwert SD, Andrews SW, Jiang Y, Serebryany V, Tan H, Kossen K, Rajagopalan PT, Misialek S, Stevens SK, Stoycheva A, Hong J, Lim SR, Qin X, Rieger R, Condroski KR, Zhang H, Do MG, Lemieux C, Hingorani GP, Hartley DP, Josey JA, Pan L, Beigelman L, Blatt LM. Preclinical characteristics of the hepatitis C virus NS3/4A protease inhibitor ITMN-191 (R7227). Antimicrob. Agents Chemother. 2008, 52, 4432-4441.

Vliegen I, Paeshuyse J, De Burghgraeve T, Lehman LS, Paulson M, Shih IH, Mabery E, Boddeker N, De Clercq E, Reiser H, Oare D, Lee WA, Zhong W, Bondy S, Pürstinger G, Neyts J. Substituted imidazopyridines as potent inhibitors of HCV replication. J. Hepatol. 2009, 50, 999-1009. 


\section{Legends to the Figures}

Figure 1. Schematic representation of the clearance-rebound experiment. During the consecutive clearance passages of $\mathrm{HCV}$ subgenomic replicon containing cells, antiviral treatment will result in inhibition of replicon replication. This in turn results in reduced levels of replicon RNA copies, that is paralleled by reduced translation of replicon encoded proteins, including the neomycinphosphotransferase gene (Neo). During the rebound passages the antiviral inhibitors are no longer present, as consequence replication can resume and subgenomic replicon containing cells are selected by the addition of G418. Only cells that express sufficient quantities of the Neo-gene encoded by the replicon will survive the selective pressure of the added G418. C= Clearence passage,

$\mathrm{R}=$ Rebound Phase.

Figure 2. Clearance of HCV RNA from replicon-containing Huh 9-13 cells following treatment with quadruple DAA combinations (i.e. 1x $\mathrm{EC}_{\mathrm{x}}$ danoprevir $+1 \mathrm{x} \mathrm{EC}_{\mathrm{x}}$ daclatasvir $+1 \mathrm{x} \mathrm{EC}_{\mathrm{x}} \mathrm{VX}-222+1 \mathrm{x} \mathrm{EC}_{\mathrm{x}}$ tegobuvir). Six consecutive clearance passages $[\mathrm{C} 1 \rightarrow \mathrm{C} 6]$ (in the presence of a fixed concentration of test compound) are followed by three consecutive rebound passages [R1 $\rightarrow R 3$ ] (with G418 pressure and without test compound). Clearance capacities of each consecutive passage in the presence of drugs but absence of $\mathrm{G} 418$ pressure are depicted o: control (no drug), $\square: \mathrm{EC}_{6}, \Delta: \mathrm{EC}_{25}, \mathrm{x}: \mathrm{EC}_{50}, \diamond: \mathrm{EC}_{75}$, *: no resistance associated mutations.

Figure 3. Clearance of HCV RNA from replicon-containing Huh 9-13 cells following treatment with single DAAs each at $4 x$ their $\mathrm{EC}_{75}$ concentration. Six consecutive clearance passages [C1 $\left.\rightarrow \mathrm{C} 6\right]$ (in the presence of a fixed concentration of test compound) are each followed by three consecutive rebound passages [R1 $\rightarrow$ R3] (with G418 pressure and without compounds). Clearance capacities of each consecutive passage in the presence of drugs but absence of G418 pressure are depicted o: control (no drug), 口: 4x EC 75 VC-222, +: 4x EC 75 daclatasvir, x: 4x EC 75 danoprevir, $\Delta: 4 x \mathrm{EC}_{75}$ tegobuvir , *: no resistance associated mutations. 
Figure 4. Clearance of HCV RNA from replicon-containing Huh 9-13 cells following treatment with triple DAA combinations (i.e. 1.33x ECx danoprevir + 1.33x ECx daclatasvir + 1.33x ECx tegobuvir) whereby $x$ can be either $6,25,50$ or 75 . Six consecutive clearance passages [C1 $\rightarrow C 6$ ] (in the presence of the inhibitors) are each followed by three consecutive rebound passages [R1 $\rightarrow R 3$ ] (with G418 pressure and without test compound). Clearance capacities of each consecutive passage in the presence of HCV inhibitors but absence of $\mathrm{G} 418$ pressure. o: control (no drug), $\square: \mathrm{EC}_{6}, \Delta: \mathrm{EC}_{25}, \mathrm{x}: \mathrm{EC}_{50}, \diamond: \mathrm{EC}_{75}$ *: no resistance associated mutations. 
Figure 1.




Figure 2.

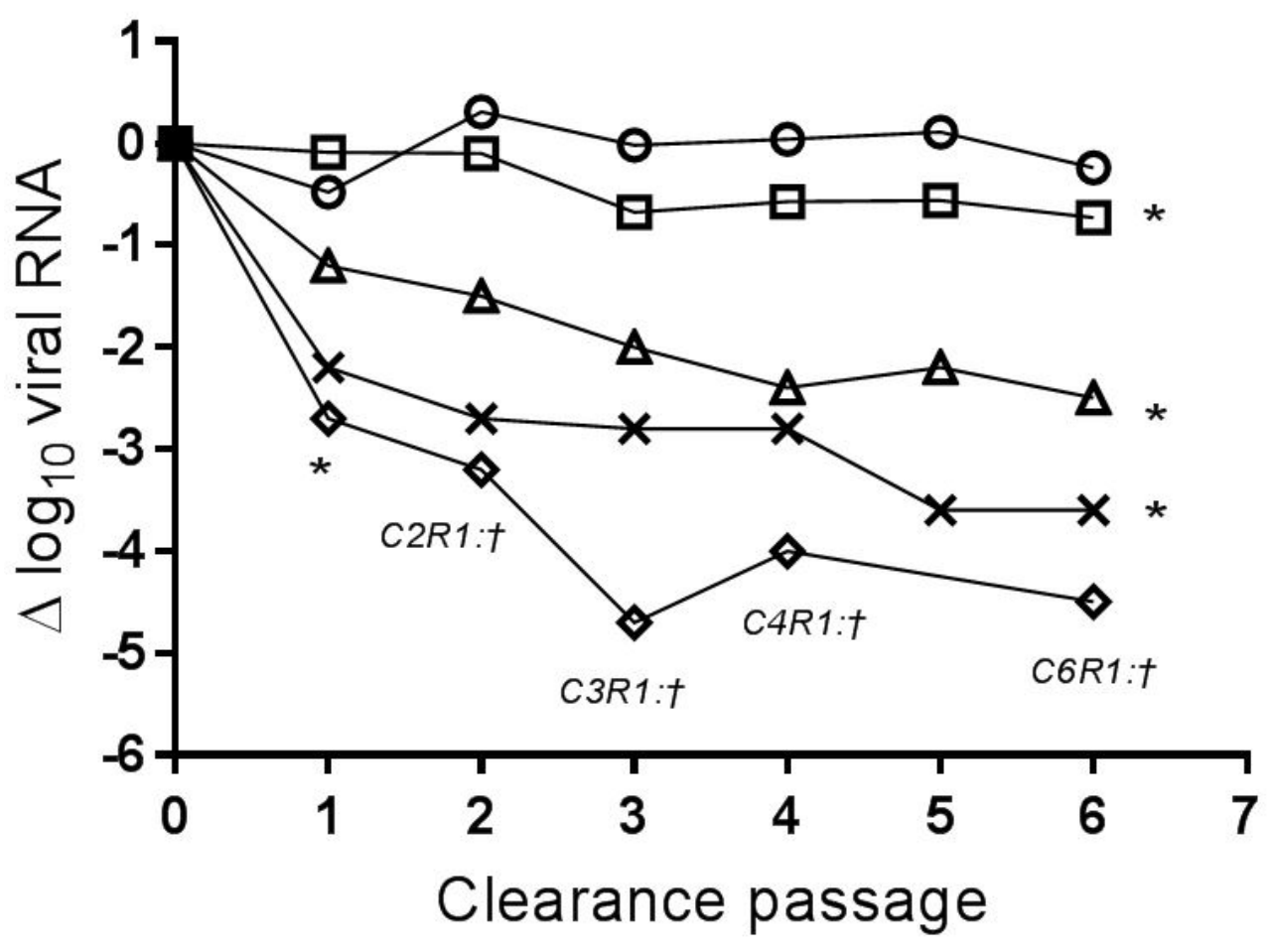


Figure 3.

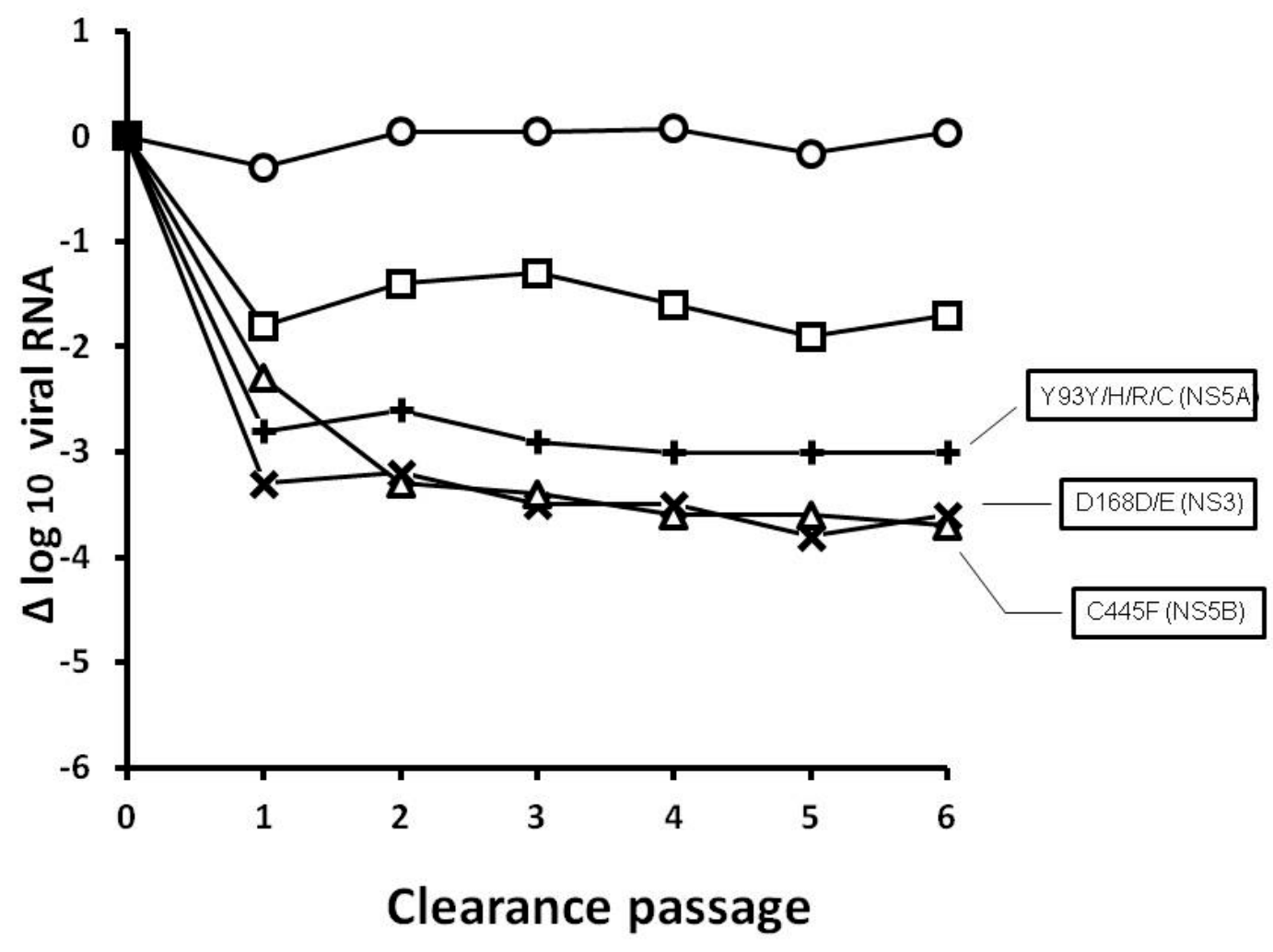


Figure 4.




Table 1. Comparison of theoretically predicted antiviral activity with the in vitro antiviral efficacy of quadruple DAA combinations or triple DAA combinations.

\begin{tabular}{|c|c|c|c|c|}
\hline \multirow[b]{3}{*}{ Treatment } & \multicolumn{4}{|c|}{ \% inhibition } \\
\hline & \multicolumn{2}{|c|}{ Quadruple DAA combinations $^{a}$} & \multicolumn{2}{|c|}{ Triple DAA combinations $^{b}$} \\
\hline & $\begin{array}{c}\text { Theoretically } \\
\text { predicted }\end{array}$ & $\begin{array}{l}\text { In vitro } \\
\text { assayed }\end{array}$ & $\begin{array}{c}\text { Theoretically } \\
\text { predicted }\end{array}$ & $\begin{array}{c}\text { In vitro } \\
\text { assayed }\end{array}$ \\
\hline $\mathrm{EC}_{75}$ & 99 & $99 \pm 0.71$ & 98 & $99 \pm 0.61$ \\
\hline $\mathrm{EC}_{50}$ & 94 & $95 \pm 5.0$ & 88 & $92 \pm 5.0$ \\
\hline $\mathrm{EC}_{25}$ & 68 & $76 \pm 19$ & 58 & $61 \pm 26$ \\
\hline $\mathrm{EC}_{13}$ & 43 & $57 \pm 25$ & 34 & $39 \pm 27$ \\
\hline $\mathrm{EC}_{6}$ & 22 & $43 \pm 24$ & 17 & $27 \pm 22$ \\
\hline
\end{tabular}

a 1x ECx danoprevir $+1 x$ ECx daclatasvir $+1 x$ ECx VX-222 + 1x ECx tegobuvir

b 1.33x ECx danoprevir + 1.33x ECx daclatasvir + 1.33x ECx tegobuvir 